\title{
Study on an amine-containing benzoxazine: Homo- and copolymerization with epoxy resin
}

\author{
L. Zhang $^{1}$, M. Wang ${ }^{2}, J . W u^{1,2^{*}}$ \\ ${ }^{1}$ Department of Mechanical and Aerospace Engineering, The Hong Kong University of Science and Technology, Clear \\ Water Bay, Kowloon, Hong Kong \\ ${ }^{2}$ Center for Engineering Materials and Reliability, Fok Ying Tung Research Institute, The Hong Kong University of \\ Science and Technology, Nansha, Guangzhou, China
}

Received 25 December 2015; accepted in revised form 23 February 2016

\begin{abstract}
An amine-containing benzoxazine (P-deta) based homopolymer and its epoxy (Ep) copolymers are investigated in this study. The structure of benzoxazine, prepared from phenol, diethylenetriamine and formalin, is confirmed by Fourier transform infrared spectroscopy (FT-IR) and nuclear magnetic resonance spectroscopy (NMR). The viscosity of P-deta is much lower than that of commercialized benzoxazine monomers. The curing profiles of P-deta with various Ep ratios are studied by deconvolution and the related mechanism is clarified. Epoxy will not increase the curing temperature of P-deta, instead, it helps P-deta overcome the conversion limitation and promotes the curing rate. The thermal and viscoelastic properties were determined and compared. The copolymer of P-deta/Ep $=50 / 50(\mathrm{wt} / \mathrm{wt}$ ) shows the highest glass transition temperature, higher than both individual homopolymers and other copolymers. Increasing benzoxazine fraction enhances modulus at room temperature but deteriorates the crosslink density. It can also enhances the char yield, but deteriorates the thermal stability of the copolymers.
\end{abstract}

Keywords: thermosetting resins, benzoxazine, epoxy resin, copolymerization

\section{Introduction}

Polybenzoxazines constitute a new generation of thermosets, which combine the thermal properties of phenolic resins with the mechanical properties and the molecular design flexibility of epoxy resin [1]. Moreover, they possess other features, such as low flammability, low water absorption, high chemical resistance and minimal shrinkage upon curing [2]. Therefore, they are considered as promising materials in IC packaging, aerospace engineering and HT-PCB industry and become one of the rare new polymers commercialized in the nearest 30 years [3]. However, several disadvantages appear in practical use. For instance, the solid phase of most benzoxazine $(\mathrm{Bz})$ monomers and the high liquefying temperature hinder their handling $[2,4]$. Adding reactive diluents is one method to decrease the operation temperature, however, simultaneously sacrifice some properties, such as flexural strength and glass transition temperature $\left(T_{\mathrm{g}}\right)$ [5]. Another method is to develop more monomers with low liquefying temperature, but only very few related researches have been reported so far $[4,6]$.

Another drawback is the low crosslink density in consequence of hydrogen bonding constraint [7]. This can be addressed by copolymerization with epoxy resin (Ep), but will lead to higher curing temperature, known as Ep dilution effect [8]. Because epoxy group is inert to oxazine ring, introducing Ep dilutes the concentration of $\mathrm{Bz}$, thus leads to higher $\mathrm{Bz}$ ringopening temperature. Besides, copolymerization with Ep happens only when phenol generates after ring-

\footnotetext{
${ }^{*}$ Corresponding author, e-mail: mejswu@ust.hk (C) BME-PT
} 
opening of Bz. Similar phenomena were also found in a main-chain type Bz/Ep binary copolymer [9]. In addition, Bz fraction should be larger than $50 \mathrm{~mol} \%$ in order to achieve copolymers with good quality. Generally, two methods can overcome the challenges. One is introducing a reactive promoter. Based on the cationic ring-opening mechanism [10], various promoters have been reported till now, among which, phenols, thiols, amines and imidazoles have attracted intensive attention since they are applied in epoxy curing as well. In 2000, phenolic resin was first selected due to its wide application in electronic packaging $[11,12]$. The material properties were significantly enhanced although the dilution effect still can be observed. Besides, Chow et al. [13] had used a phenol monomer, 4,4'-thiodiphenol to study the curing behavior of a Bz/Ep/phenol ternary system. In 2005, Rao and Pathak [14] managed to use a phenalkamine to prevent the dilution effect through prior reaction between phenalkamine and Ep at room temperature before mixing with Bz. More recently, Grishchuk et al. [15] selected aliphatic, cycloaliphatic and aromatic amines to fabricate the Bz/Ep copolymers. They found that amines, which are both reactive to Bz and Ep, can efficiently promote the copolymerization. In addition, they also found that the promotion effect was influenced by both amine type and amount. The related mechanism was later clarified by Sun et al. [16], who purposed that the promotion effect is based on the basicity of amines. Moreover, Ambrožič et al. [17] used o-dianisidine to prepare novel Ep-Bz emulsions for water-based coatings to ensure a highly cross-linked structure. Meanwhile, Zhao et al. [18] and Wang et al. [19] studied a Bz/Ep/imidazole ternary system, through which the curing steps were optimized to motivate reaction-induced phase separation so that toughness can be enhanced. Ručigaj et al. [20] had implemented a comprehensive study on the curing Bz with many accelerators with different functional groups. Based on the conversion prediction, 4,4'-thiodiphenol is considered as the most effective accelerator among them. However, the solid forms of $\mathrm{Bz}$ and many additives generally require pretreatment before polymerization. Another method is to incorporate reactive groups, such as amine, onto the $\mathrm{Bz}$ structures. But, only few studies have been reported until now due to relatively tedious preparation procedure [21, 22].
In this research, we attempted to apply a low viscous amine-containing $\mathrm{Bz}$ to facilitate processing, promote polymerization as well as achieve homo- and copolymers with good performance. Meanwhile, we also tried to find its differences from a $\mathrm{Bz} / \mathrm{Ep} / \mathrm{amine}$ ternary system. The present study deals with the synthesis of the Bz, preparation of its homopolymer and copolymers, investigation of the curing behavior as well as evaluation of the thermal and mechanical properties.

\section{Experimental}

\subsection{Materials}

All reagents for synthesis are commercially available and used as received. Phenol (99\%), bisphenol A (99\%), aniline (99\%) and deuterated chloroform (with $0.03 \mathrm{vol} \%$ of tetramethylsilane as reference) were purchased from Aladdin Reagent (Shanghai) Co. Ltd., Shanghai, China. Formaldehyde solution (40 wt \%), paraformaldehyde (96\%), soldium sulfate anhydrous (99\%), diethylenetriamine (DETA) $(99 \%)$ and potassium bromide (99.5\%) were acquired from SigmaAldrich, St. Louis, U.S. Sodium carbonate anhydrous (99.9\%) and dichloromethane (99.8\%) were obtained from VWR, Radnor, U.S. A bifunctional diglycidyl ether bisphenol A (DGEBA) based epoxy resin (E51, epoxy equivalent weight: 185-208 g/ep.) was supported by Wuxi resin company, Wuxi, China.

\subsection{Sample preparation methods}

\subsubsection{Synthesis of $\mathrm{N}, \mathrm{N}$-bis[2-(3,4-dihydro-2H-}

1,3-benzoxazin-3-yl)ethyl] amine (P-deta)

The synthesis method is a modification of preparing a main-chain type benzoxazine polymer reported elsewhere [23, 24]. Specifically, phenol (18.8 g, $0.2 \mathrm{~mol})$ was dissolved in dichloromethane $(50 \mathrm{~mL})$ in a $250 \mathrm{~mL}$ flask before adding DETA (10.3 g, $0.1 \mathrm{~mol})$. The mixture was then kept below $10^{\circ} \mathrm{C}$ in an ice bath and stirred when formaldehyde solution was added dropwise. The molar ratio is maintained at phenol:DETA:formaldehyde $=2: 1: 4$. The mixture reacted overnight and the solution gradually changed from colorless to yellow. Later on, the mixture was washed by $1 \mathrm{~N}$ sodium carbonate solution and deionized water, respectively. After being dried by sodium sulfate anhydrous, the obtained liquid was dried under vacuum at room temperature to evaporate solvent before obtaining a homogeneous yellow viscous liquid (Yield $=85 \%)$. 


\subsubsection{Synthesis of benzoxazine precursor (BA-a)} For comparison, N-phenyl bisphenol A based benzoxazine precursor (BA-a) was prepared according to the solventless method patented by Ishida [25]. (Yield $>90 \%$ )

\subsubsection{Preparation of P-deta homopolymer and P-deta/Ep copolymers}

P-deta was mixed with Ep under room temperature at ratios of P-deta:Ep $=100 / 0,75 / 25,50 / 50$ and 25/75 (wt/wt) before intensively stirring for $5 \mathrm{~min}$. After degassing at $50^{\circ} \mathrm{C}$ for $10 \mathrm{~min}$, all of them were stepwise cured at $125^{\circ} \mathrm{C} / 1 \mathrm{~h}, 150^{\circ} \mathrm{C} / 1 \mathrm{~h}$ and $180^{\circ} \mathrm{C} / 4 \mathrm{~h}$.

\subsection{Characterization methods}

The Fourier transform infrared spectroscopy (FT-IR) spectra of P-deta were recorded by a Bruker TENSOR27 FT-IR spectrometer (Bruker Corporation, Karlsruhe, Germany) with 16 scans at a $4 \mathrm{~cm}^{-1}$ resolution. It was also applied to monitor the curing process underwent isothermal treatment $(100,125$, $\left.150,175,200^{\circ} \mathrm{C}\right)$. Potassium bromide $(\mathrm{KBr})$ pellet technique was used.

The structures of the compounds were verified by nuclear magnetic resonance spectroscopy (NMR) using a Varian Mercury VX 300 NMR, Varian Inc., Palo Alto, U.S. The characterization was conducted at room temperature using deuterated chloroform $\left(\mathrm{CDCl}_{3}\right)$ as solvent and tetramethylsilane (TMS) as internal standard.

The viscosity of the precursors was recorded by a rheometer, (AR2000ex, TA Instrument, New Castle, U.S.) under steady shear mode. The rotation frequency was set at $1 \mathrm{~s}^{-1}$ with a temperature range from room temperature to above gel point and a heating rate of $2{ }^{\circ} \mathrm{C} / \mathrm{min}$. The polymerization processes of all samples were monitored by a differential scanning calorimeter (DSC), (Q200, TA Instrument, New Castle, U.S.). All samples with 3-5 mg were sealed in aluminum pans before measurement. The thermograms were obtained at a heating rate of $10^{\circ} \mathrm{C} / \mathrm{min}$ from room temperature to $300^{\circ} \mathrm{C}$ under nitrogen atmosphere.

The viscoelastic properties of all samples were investigated by a dynamic mechanical analyzer (DMA), (Q800, TA Instrument, New Castle, U.S). The specimen dimension was $10 \times 70 \times 2 \mathrm{~mm}$. The measurement was performed in a three-point bending mode with $50 \mathrm{~mm}$ span length at a frequency of $1 \mathrm{~Hz}$ with a strain value of $0.1 \%$ and a heating rate of $2{ }^{\circ} \mathrm{C} / \mathrm{min}$. The glass transition temperature $\left(T_{\mathrm{g}}\right)$ was determined by the peak temperature of loss factor $(\tan \delta)$.

The thermal stability and char yield are characterized through a thermogravimetric analyzer (TGA), (Q50, TA Instrument, New Castle, U.S.). The test was performed under nitrogen atmosphere at a heating rate of $10^{\circ} \mathrm{C} / \mathrm{min}$.

\section{Results and discussion}

\subsection{Characterization of P-deta}

The structure of P-deta was first studied by FT-IR, as shown in Figure 1. The reaction scheme is plotted in Figure 1 as well. The formation of oxazine ring was evidenced by the peak at $930 \mathrm{~cm}^{-1}(1,2$-substituted benzene with oxazine ring attaching), the sy4mmetric and asymmetric stretching of $\mathrm{C}-\mathrm{O}-\mathrm{C}$ at 1034 and $1226 \mathrm{~cm}^{-1}$, and the peak at $1150 \mathrm{~cm}^{-1}$ which indicates the formation of $\mathrm{C}-\mathrm{N}-\mathrm{C}$ bond. The peak at 1489 and $756 \mathrm{~cm}^{-1}$ prove the presence of an ortho-substituted benzene ring. Since hydroxyl group is removed by base washing, the shoulder peak near $3300 \mathrm{~cm}^{-1}$ is assigned to the heavily overlapped hydrogen bonded amino group. These results confirm the formation of the P-deta.

NMR was further applied to characterize the structures, the results of which are shown in Figure 2. Specifically, the characteristic proton resonances at 4.0 and $4.9 \mathrm{ppm}$ are corresponding to the $\mathrm{Ar}-\mathrm{CH}_{2}-\mathrm{N}$ and $\mathrm{N}-\mathrm{CH}_{2}-\mathrm{O}$ structures of oxazine ring, respectively. The resonances at $6.8-7.2 \mathrm{ppm}$ are assigned to the protons on aromatic rings. The peaks in the range of 3.7-3.9 ppm were assigned to $\mathrm{Ar}-\mathrm{CH}_{2}-\mathrm{N}$ and $\mathrm{N}-\mathrm{CH}_{2}-\mathrm{N}$ structures, which has been reported in other literatures as well [16, 23, 24]. A resonance chemical shift at $3.5 \mathrm{ppm}$ confirms the formation of $\mathrm{N}-\mathrm{H}$. The aliphatic groups of amines are confirmed by the resonances at $2.7-2.9 \mathrm{ppm}$, respectively. The

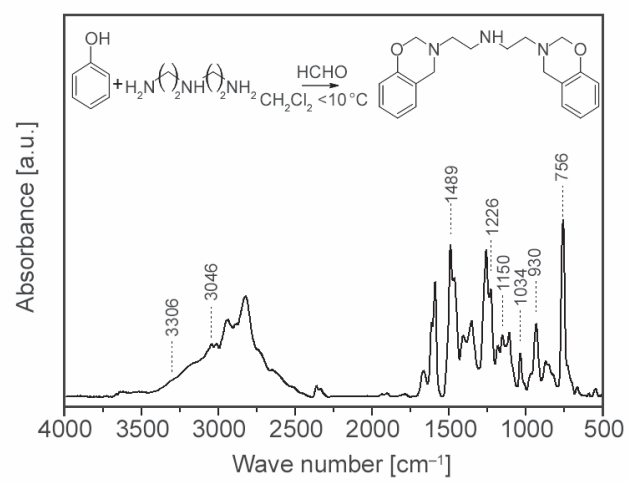

Figure 1. FT-IR spectrum of P-deta 


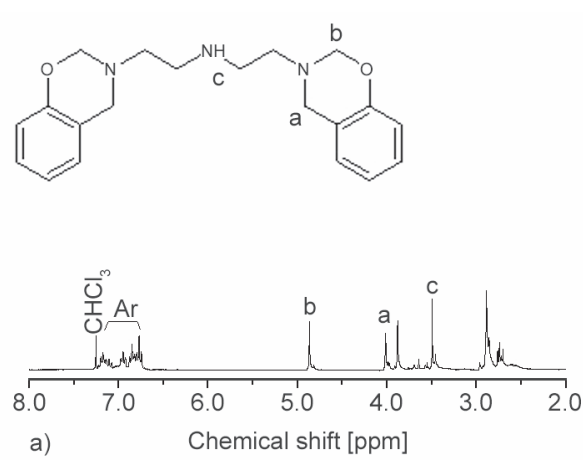

Figure 2. (a) ${ }^{1} \mathrm{H}-\mathrm{NMR}$ and (b) ${ }^{13} \mathrm{C}-\mathrm{NMR}$ results of P-deta

product was also characterized by ${ }^{13} \mathrm{C}-\mathrm{NMR}$ (Figure $2 \mathrm{~b}$ ). The characteristic oxazine rings appear at 83 and $50 \mathrm{ppm}$. The peaks at $115-130$ and $154 \mathrm{ppm}$ indicate the phenyl carbons. The chemical shift at $158 \mathrm{ppm}$ is assigned to the ring-opened $\mathrm{Bz}$ structures. The chemical shift at $76 \mathrm{ppm}$ confirms the existence of triazine structure. The peaks between 50 $60 \mathrm{ppm}$ indicate various $\mathrm{Ar}-\mathrm{CH}_{2}-\mathrm{N}$ and $\mathrm{N}-\mathrm{CH}_{2}-\mathrm{N}$ structures, which is consistent with the ${ }^{1} \mathrm{H}-\mathrm{NMR}$ results.

Before polymerization, the viscosity of P-deta and P-deta/Ep mixture was investigated (Figure 3). A typical benzoxazine monomer, BA-a, was used for reference. Comparing with $\mathrm{BA}-\mathrm{a}$, the viscosity of $\mathrm{P}-$ deta is very low $(<1 \mathrm{~Pa} \cdot \mathrm{s})$ under room temperature. This makes it suitable for some specific processing methods, like resin transfer molding (RTM). The storage condition can significantly change the viscosity of P-deta. It can be seen that the viscosity of P-deta increased to above $2000 \mathrm{~Pa} \cdot \mathrm{s}$ after being kept under room temperature for more than 1.5 month, which is due to the ring-opening of benzoxazine caused by amines. Therefore, low temperature storage is required for the resin. Here, the gel point is defined as the temperature when viscosity suddenly increases to above $100 \mathrm{~Pa} \cdot \mathrm{s}$, and the processing window is de-

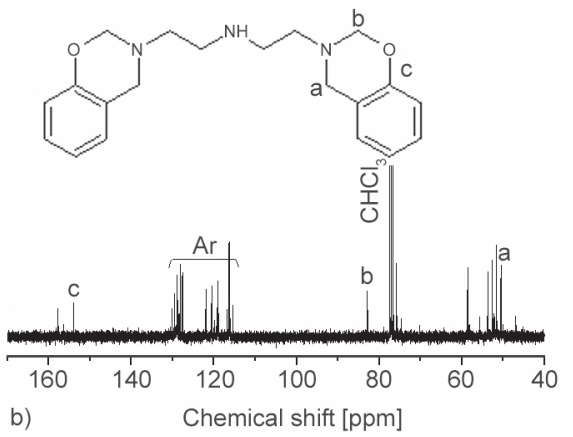

fined as the temperature range, within which the viscosity of the sample is lower than $100 \mathrm{~Pa} \cdot \mathrm{s}$. The gel point of P-deta/Ep $(50 / 50, \mathrm{wt} / \mathrm{wt})$ mixture is at $150^{\circ} \mathrm{C}$, while it is more than $200^{\circ} \mathrm{C}$ where BA-a reaches its gel point. The gel point of pure $\mathrm{P}$-deta cannot be determined due to the extremely low viscosity when heated P-deta up to $100^{\circ} \mathrm{C}$. The processing window of P-deta/Ep $(50 / 50, \mathrm{wt} / \mathrm{wt})$ mixture is larger than $125^{\circ} \mathrm{C}$ (R.T. to $\left.150^{\circ} \mathrm{C}\right)$.

\subsection{Polymerization of P-deta and P-deta/Ep mixtures}

DSC and FT-IR were applied to investigate the polymerization of P-deta and P-deta/Ep mixtures of various ratios. Comparing with the reported mono-curing peak of BA-a [15], multi-curing peaks can be found in P-deta, which can be assigned to several different reactions. In fact, it becomes more complicated when Ep is introduced. In order to clarify the possible reaction of each peak, the curing profiles of P-deta and P-deta/Ep mixtures have been deconvoluted. The DSC profiles and peaks achieved by deconvolution are summarized in Figure 4. Specifically, three peaks appear during P-deta homopolymerization which, from low to high temperature, are related to amine catalyzed $\mathrm{Bz}$ ring-opening, Bz thermal-in-
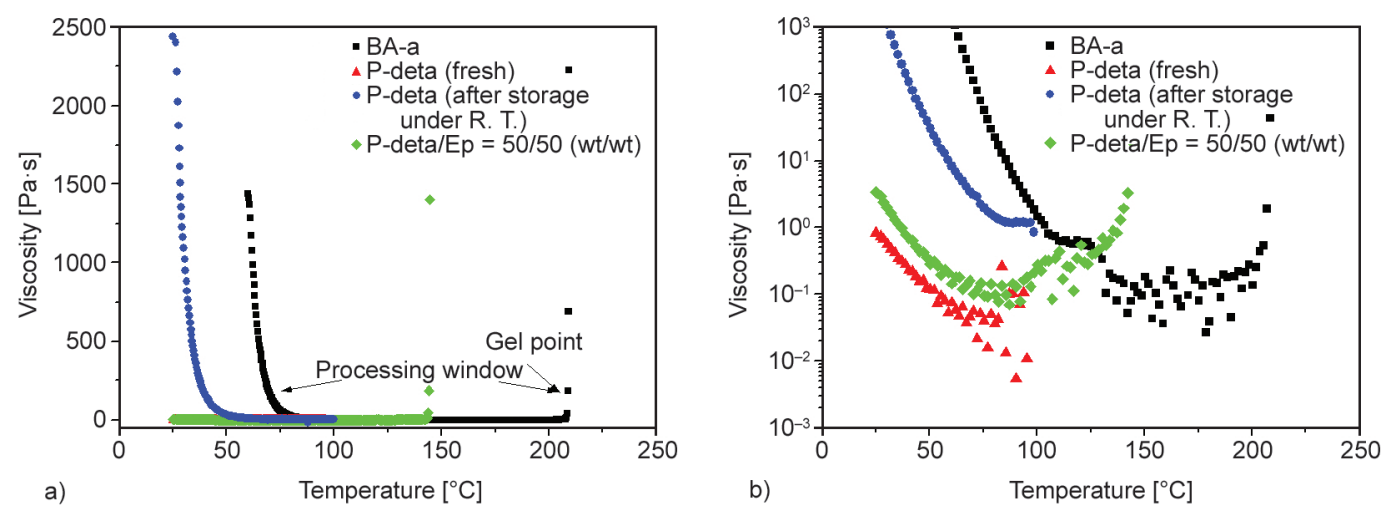

Figure 3. (a) Prcessing window and (b) viscosity of P-deta, P-deta/Ep (50/50, wt/wt) and BA-a 

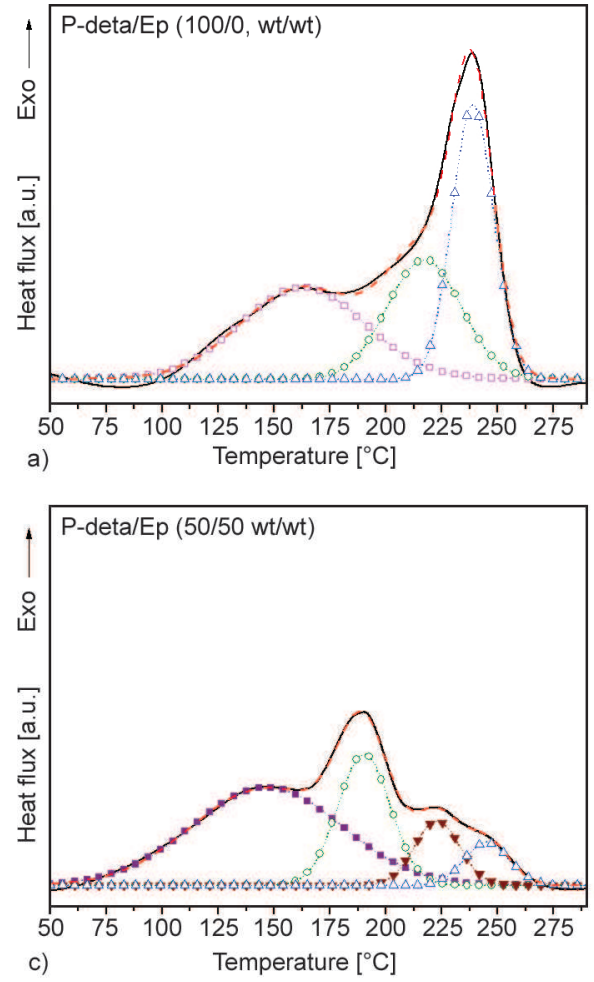
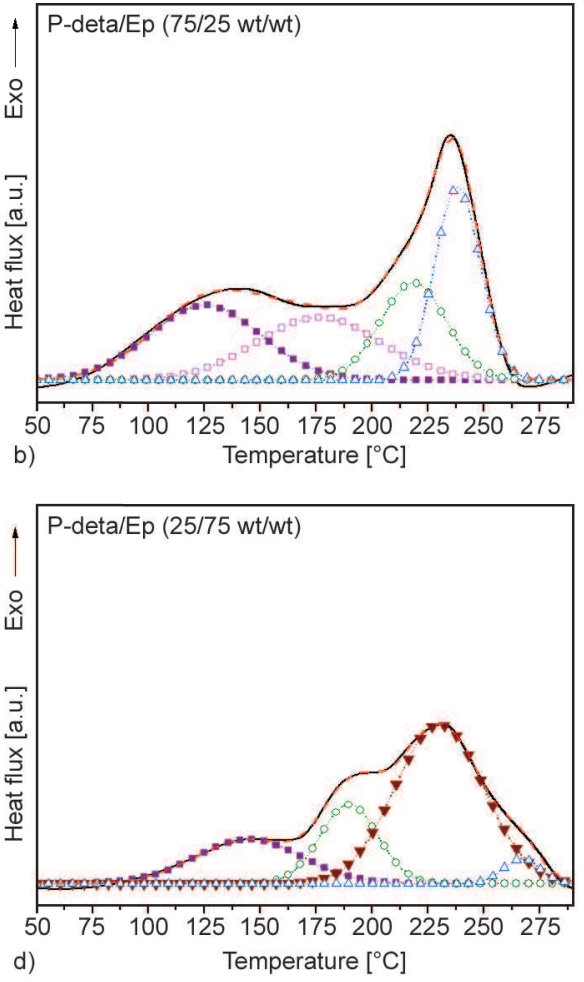

Figure 4. DSC profiles (solid line) and deconvolution peaks (dash line) of P-deta with different amount of Ep. (P-deta/Ep = (a) $100 / 0$, (b) $75 / 25$, (c) 50/50 and (d) 25/75, wt/wt). Peak assignment: ( $\square$ ) amine-catalyzed Bz ring-opening; ( $)$ amine-Ep ring-opening; ( $\circ)$ Bz ring-opening; $(\triangle)$ intermediate conversion; $(\boldsymbol{\nabla})$ hydroxyl-Ep ring-opening.

duced polymerization and conversion of amine-Bz intermediate to final structure [16], respectively. It is worth to note that, intermediate conversion can happen at the temperature either higher [16] or lower [15] than the origin, it is higher than Bz polymerization in this case. When mixed with Ep at P-deta/Ep = $75 / 25$ (wt/wt), one additional peak can be found at low temperature range. It can be assigned to the reaction between amine and Ep, which takes place at relatively low temperature. This reaction can also be proved by the shift of onset temperature from $90^{\circ} \mathrm{C}$ to $60-70^{\circ} \mathrm{C}$ as long as Ep was added. Further increasing Ep amount to $50 \mathrm{wt} . \%$ (Figure 4c) leads to an increase in intensity at $190^{\circ} \mathrm{C}$ but a decrease around $240^{\circ} \mathrm{C}$. Although the peak at $190^{\circ} \mathrm{C}$ is close to the second peak position in Figure $4 \mathrm{~b}$, it is impossible due to large consumption of amine during amine-Ep reaction. In fact, it is probably caused by $\mathrm{Bz}$ ring-opening, which is promoted by the hydroxyl group due to the same reaction [26]. The rest small amount of excess Ep can react with phenol (generated after $\mathrm{Bz}$ ring-opening) or hydroxyl group, which results in the third peak in Figure 4c. The intensity is obviously enhanced in Figure $4 \mathrm{~d}$ due to the increase of Ep to $75 \mathrm{wt} \%$. In addition, the last tiny peaks in both Figure 4c and Figure 4d may be as- signed to the conversion of intermediate. Since the reactions between amine-Bz and amine-Ep are partially overlapped, it is possible that a small portion of amine reacts with $\mathrm{Bz}$ when simultaneously most of it reacts with Ep. It is worth to mention that, by prior reaction between Ep and amine (in $\mathrm{Bz}$ ), the dilution effect caused by Ep can be eliminated, based on our study.

The possible reactions take place during the polymerization were further investigated through FT-IR, as shown in Figure 5. The samples are partially cured under different isothermal conditions. Concerning Pdeta (Figure $5 \mathrm{a}$ and $5 \mathrm{~b}$ ), the peak at $930 \mathrm{~cm}^{-1}$, which represents the out-of-plane bending of benzene ring with oxazine ring being attached, dramatically decreases after heating at $125^{\circ} \mathrm{C}$ for 1 hour and almost disappeared after heating at $150^{\circ} \mathrm{C}$. The decrease of overlapped peaks near $3300 \mathrm{~cm}^{-1}$ proved the aminepromoted ring-opening process. The intensity increase around $3450 \mathrm{~cm}^{-1}$ is attributed to the hydroxyl group, which formed after $\mathrm{Bz}$ ring-opening. Moreover, the intensity decrease at $1489 \mathrm{~cm}^{-1}$, which represents the consumption of Bz. However, no observable intensity change can be found in BA-a even heated at $175^{\circ} \mathrm{C}$ for 1 hour (Figure $5 \mathrm{c}, 942 \mathrm{~cm}^{-1}$ ). It is until $200^{\circ} \mathrm{C}$ that most of the BA-a precursors un- 

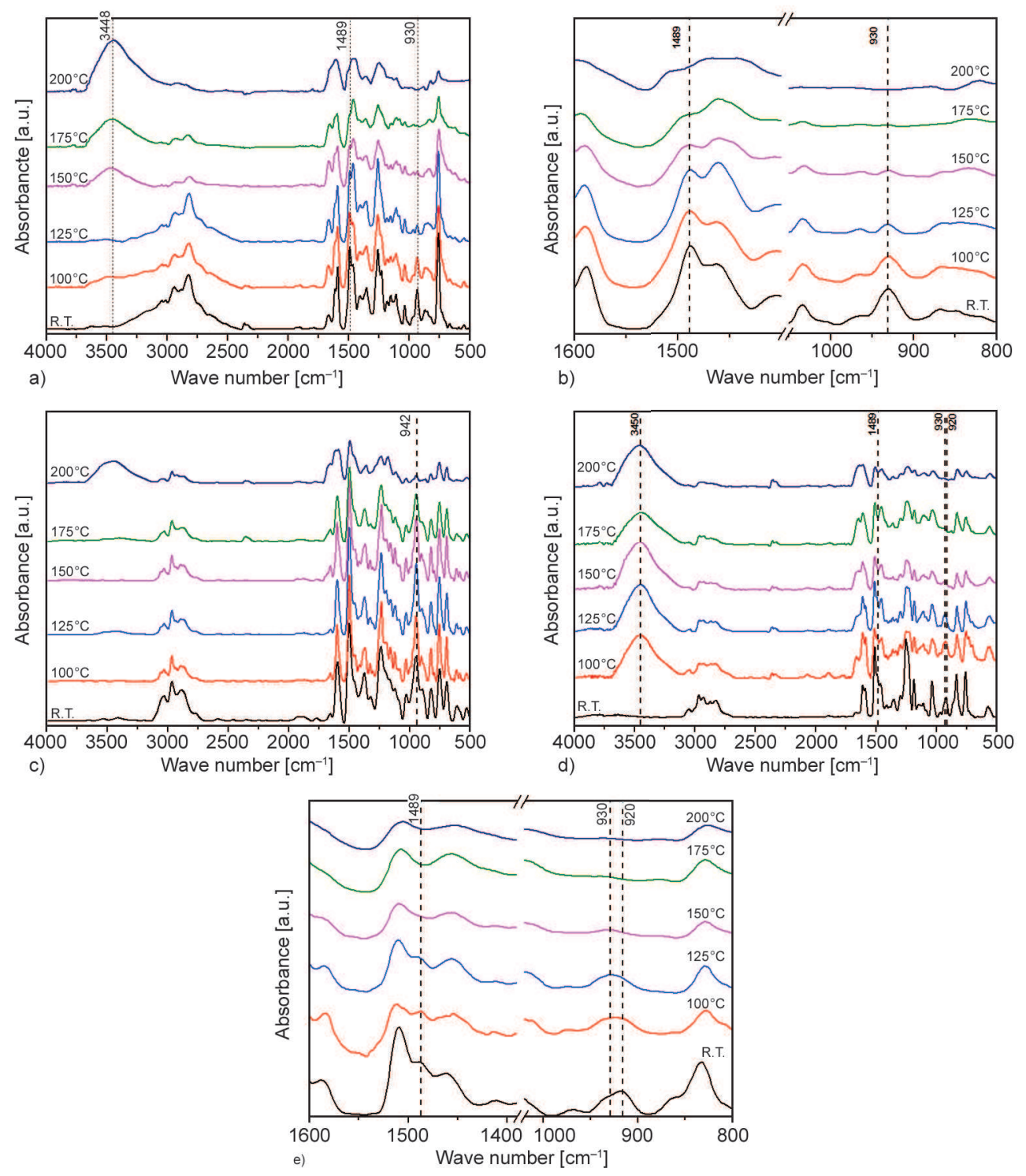

Figure 5. FT-IR spectra of (a) and (b) P-deta, (c) BA-a and (d) and (e) P-deta/Ep $=50 / 50$ (wt/wt) after isothermally cured at $100,125,150,175$ and $200^{\circ} \mathrm{C}$ for 1 hour, respectively

dergo ring-opening polymerization. The curing process changes when Ep was introduced. The FTIR results of $\mathrm{P}$-deta/Ep $=50 / 50(\mathrm{wt} / \mathrm{wt})$ are summarized in Figure 5d. Comparing with P-deta precursor, a conspicuous peak at $3450 \mathrm{~cm}^{-1}$ can be found even heated at $100^{\circ} \mathrm{C}$ for 1 hour, which is attributed to the hydroxyl groups generated after the reaction between amine and Ep. This is also proved by the decrease at $920 \mathrm{~cm}^{-1}$ in Figure 5e, which represents the epoxy ring. Besides, the peaks at $1489 \mathrm{~cm}^{-1}(1,2-$ substituted benzene ring) and $930 \mathrm{~cm}^{-1}$ (out-of-plane benzene ring with oxazine attaching) disappear at $150^{\circ} \mathrm{C}$, which confirmed the ring-opening of oxazine as well. These results are consistent with the DSC peaks observed in Figure 4.
The partially cured polymers under isothermal conditions are examined by DSC as well (Figure 6). The degree of conversion $(\alpha)$ in Figure $6 \mathrm{~d}$ is calculated through Equation (1), based on the DSC thermograms:

$\alpha[\%]=\left(1-\frac{\Delta H_{\mathrm{p}}}{\Delta H_{0}}\right) \cdot 100$

where $\Delta H_{\mathrm{p}}$ is the curing enthalpy change of the partially cured samples and $\Delta H_{0}$ is the curing enthalpy change of the original samples. Initially, the conversion rate of $\mathrm{P}$-deta is much faster than BA-a, due to the amine-promoted ring-opening reaction. However, the degree of conversion reached a limit and no further progress was observed. Comparing with Fig- 

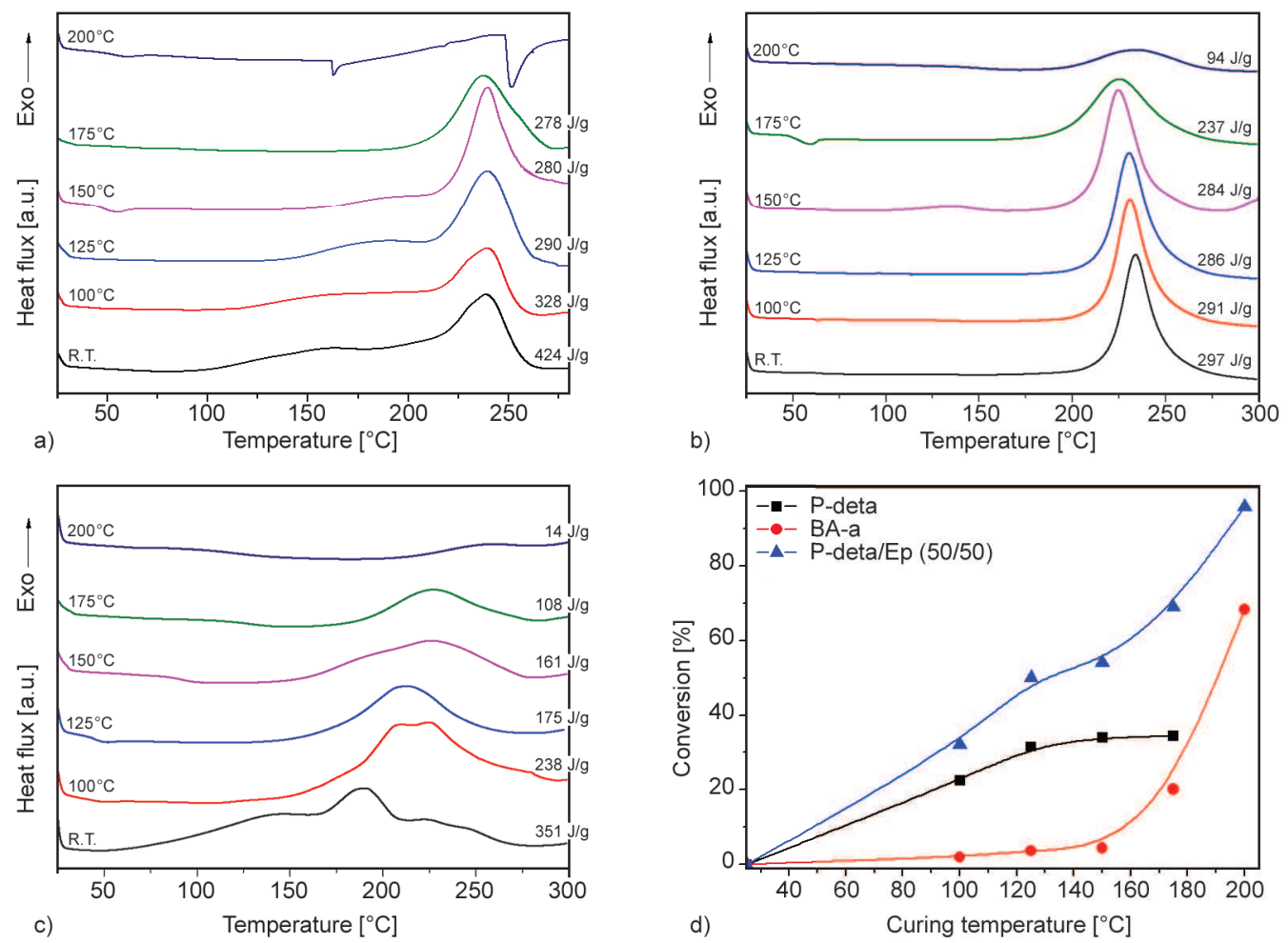

Figure 6. DSC thermograms of (a) P-deta, (b) BA-a, (c) P-deta/Ep $=50 / 50$ (wt/wt) and (d) conversion based on enthalpy change after isothermal curing at $100,125,150,175$ and $200^{\circ} \mathrm{C}$ for 1 hour, respectively

ure 4, the residue peak in Figure $6 \mathrm{a}$ at above $150^{\circ} \mathrm{C}$ is assigned to intermediate conversion. It cannot be the $\mathrm{Bz}$ ring-opening, otherwise, the conversion (Figure 6d) will be more like a autocatalytic process, similar to BA-a, rather than reached a limit. Copolymerization of Ep and P-deta changes the reactions, thus conversion will not be confined by the equilibrium, as shown in Figure $6 \mathrm{c}$ and $6 \mathrm{~d}$. A mixture of $\mathrm{P}$ $\operatorname{deta} / \mathrm{Ep}=50 / 50(\mathrm{wt} / \mathrm{wt})$ can almost be thoroughly cured after heating at $200^{\circ} \mathrm{C}$ for 1 hour. Besides, the conversion rate of $\mathrm{P}-\mathrm{deta} / \mathrm{Ep}$ mixture is higher than P-deta and BA-a. This is attributed to the hydroxyl group generated from the reaction between epoxide and amine, which can promote the ring-opening polymerization of $\mathrm{Bz}[26]$.

\subsection{Thermal and viscoelastic properties of P-deta and its epoxy alloy}

The storage moduli and loss factors of all samples are measured through DMA, and the results are shown in Figure 7. The important data is summarized in Table 1. It is worth to note that, a P-deta/Ep= 25/75 (wt/wt) copolymer, beyond the mixing limit $(\mathrm{Bz}>50 \%)$ of general Bz/Ep binary system [8], had been successfully prepared with good mechanical properties. This is due to the reaction between amine in P-deta and Ep. Besides, amine can increase the

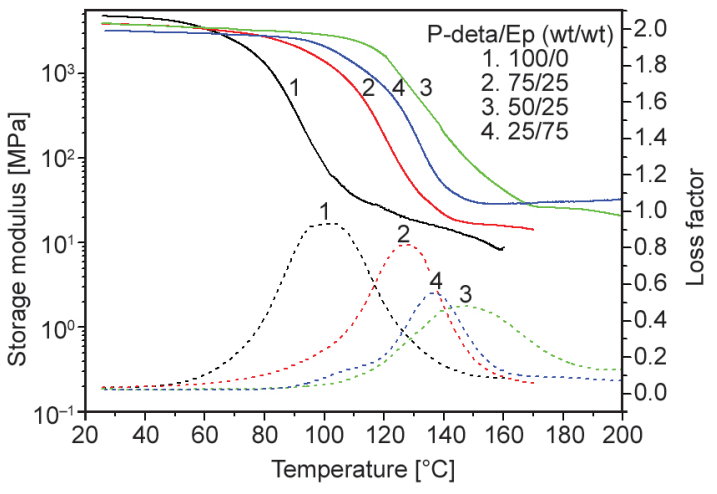

Figure 7. Storage moduli $\left(E^{\prime}\right)$ and mechanical loss factors $(\tan \delta)$ as a function of temperature for P-deta/Ep mixtures at various ratios

hydrogen bond density, thus enhances the storage modulus at room temperature of P-deta homopolymer $(4.8 \mathrm{GPa})$. The modulus of copolymers decreases with the increase of Ep fraction. This is due to the concentration of hydrogen bond density of $\mathrm{Bz}$ decreases when Ep is incorporated. Moreover, since glass state stiffness is related to the resistance of molecular segments motion, the DGEBA structure appears less able to store elastic energy, which leads to a lower modulus than $\mathrm{Bz}$ [9]. The peak temperature of loss factor is applied to determine the $T_{\mathrm{g}}$ of the samples. Unlike modulus, the $T_{\mathrm{g}}$ of P-deta homopolymer is relatively low $\left(100^{\circ} \mathrm{C}\right)$. The highest $T_{\mathrm{g}}$ at $147^{\circ} \mathrm{C}$ 
is obtained in a P-deta/Ep (50/50, wt/wt) copolymer, which is $47^{\circ} \mathrm{C}$ higher than P-deta homopolymer and $17^{\circ} \mathrm{C}$ higher than the reported Ep (cured by DETA) [15]. It is interesting to find that the result is quite different from the $\mathrm{Bz} / \mathrm{Ep} / \mathrm{amine}$ ternary copolymers, the $T_{\mathrm{g}}$ of which is between the $\mathrm{Bz}$ and Ep homopolymers or worse $[15,27]$. In fact, it is more close to $\mathrm{Bz} / \mathrm{Ep}$ binary copolymers, which has a $T_{\mathrm{g}}$ higher than both homopolymers at certain mixing ratios [10]. Specifically, at low Ep fraction, the polymer network is enhanced by Ep-amine and Ep-hydroxyl reactions. When Ep amount is in excess, it acts as a plasticizer to deteriorate the mechanical properties. The mono-peak of $\tan \delta$ showed that no apparent phase separation can be found, which is consistent with other Bz/Ep binary systems [8]. The height and width of $\tan \delta$ are summarized in Table 1 . The 50/50 copolymer shows the lowest $\tan \delta$ height, which indicates strongest segmental restriction. The FWHM (full width at half mount) of $\tan \delta$ implies the variety of structure species. Although no certain trend of FWHM can be observed among all samples, the FWHM of 50/50 sample shows the largest FWHM value $\left(43^{\circ} \mathrm{C}\right)$, which indicates the most various structures in the polymer.

Based on the statistical theory on rubbery elasticity [28], the crosslink density can be estimated through Equation (2) by obtaining the elastic modulus in rubbery state:

$v=\frac{E_{\mathrm{R}}^{\prime}}{3 \varphi R T}$

where $v$ is the crosslink density, $R$ is the gas constant and $\varphi$ equals to 1 for ideal rubber. In this experiment, the elastic modulus $E_{\mathrm{R}}^{\prime}$ is selected at the absolute temperature $T$ equals to $T_{\mathrm{g}}+30^{\circ} \mathrm{C}$. It is worth to note that, this equation is only valid in light crosslink polymers since high crosslink density results in a failure of Gaussian chain assumption [29, 30]. Therefore, an empirical Nielsen equation (Equation (3)),

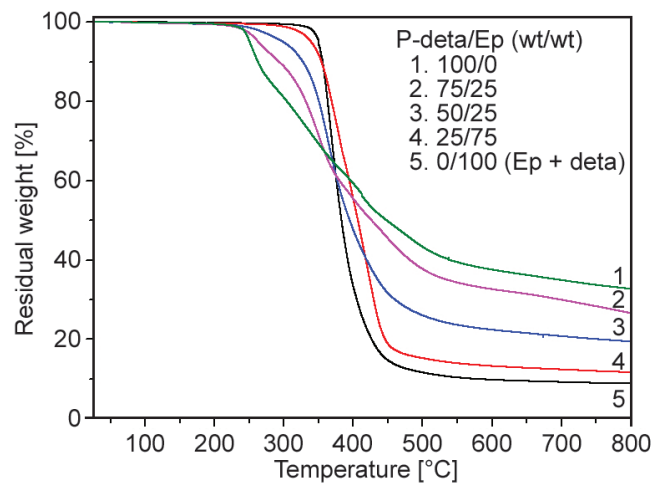

Figure 8. TGA thermograms of P-deta and P-deta/Ep polymers

which shows better results among high crosslink structures, is also selected here [31]:

$\lg \left(\frac{E_{\mathrm{R}}^{\prime}}{3}\right)=7+293 v$

The calculated results based on both methods are concluded in Table 1. Specifically, the crosslink density of P-deta is very low, which is 1790 and $2660 \mathrm{~mol} / \mathrm{m}^{3}$ based on different calculation methods. Increasing Ep fraction was found to enhance the crosslink density of the copolymers, which is consistent with the trend of Bz/Ep binary copolymers [8] but different from some Bz/Ep/amine ternary copolymers [14, 27]. The thermal stability of all samples was studied by TGA (Figure 8) and the results have been summarized in Table 1. The decomposition temperature of P-deta homopolymer is similar to MCBP BA-tepa [24] and P-ad6 [32]. The similar aliphatic diamine structure is probably the dominant cause of the initial decomposition. Incorporation of Ep enhances the thermal stability of the copolymers. This can be explained by the increase in crosslink density when increasing the Ep fraction. Meanwhile, the residual weight after heating at $800^{\circ} \mathrm{C}$ becomes lower when less $\mathrm{Bz}$ is introduced into the copolymers. The decrease in char yield percentage is quite consistent

Table 1. Viscoelastic and thermal properties of P-deta and P-deta/Ep polymers

\begin{tabular}{|c|c|c|c|c|c|c|c|c|c|}
\hline \multirow{2}{*}{$\begin{array}{c}\text { Sample } \\
\text { (P-deta/Ep, wt/wt) }\end{array}$} & \multirow{2}{*}{$\begin{array}{c}T_{\mathrm{g}} \\
{\left[{ }^{\circ} \mathrm{C}^{\mathrm{a}}\right.}\end{array}$} & \multicolumn{2}{|c|}{$\begin{array}{c}E^{\prime} \\
{[\mathrm{MPa}]}\end{array}$} & \multicolumn{2}{|c|}{$\begin{array}{c}\text { Crosslink density } \\
{\left[\cdot 10^{3} \mathrm{~mol} / \mathrm{m}^{3}\right]}\end{array}$} & \multicolumn{2}{|c|}{ Loss factor } & \multirow{2}{*}{$\begin{array}{l}T_{5 \%} \% \\
{\left[{ }^{\circ} \mathbf{C}\right]^{b}}\end{array}$} & \multirow{2}{*}{$\begin{array}{l}\text { Char } \\
\text { yield } \\
{[\%]^{\mathrm{c}}}\end{array}$} \\
\hline & & RT & $T_{\mathrm{g}}+30^{\circ} \mathrm{C}$ & $\begin{array}{c}\text { Rubber } \\
\text { elasticity }\end{array}$ & $\begin{array}{l}\text { Nielsen's } \\
\text { equation }\end{array}$ & $\begin{array}{c}\text { FWHM } \\
{\left[{ }^{\circ} \mathrm{C}\right]}\end{array}$ & Height & & \\
\hline $100 / 0$ & 100 & 4785 & 18 & 1.79 & 2.66 & 37 & 0.93 & 251 & 33 \\
\hline $75 / 25$ & 128 & 3876 & 16 & 1.49 & 2.48 & 30 & 0.82 & 263 & 27 \\
\hline $50 / 50$ & 147 & 3928 & 26 & 2.32 & 3.20 & 43 & 0.43 & 299 & 20 \\
\hline $25 / 75$ & 137 & 3220 & 29 & 2.64 & 3.36 & 26 & 0.55 & 339 & 12 \\
\hline $0 / 100^{d}$ & $130^{\mathrm{e}}$ & $2690^{\mathrm{e}}$ & $33^{\mathrm{e}}$ & $3.06^{\mathrm{e}}$ & 3.55 & - & - & 350 & 9 \\
\hline
\end{tabular}

${ }^{a}$ peak temperature of $\tan \delta$; ${ }^{b}$ temperature at which $5 \%$ weight loss is monitored; ${ }^{c}$ weight residue at $800{ }^{\circ} \mathrm{C}$; ${ }^{\mathrm{d}} \mathrm{Ep}$ was cured by DETA; ${ }^{\mathrm{e}}$ data obtained from reference [15]. 
with the decrease in benzoxazine fraction. Apparently, rather than crosslink density, Bz fraction is more dominant to determine the char yield of the copolymers.

In summary, comparing with a Bz monomer (P-ad6 in [32]) and a main-chain type Bz polymer (BA-tepa in [24]) of similar molecular structures, P-deta homopolymer exhibits higher modulus under room temperature than both while thermal stability and char yield in-between. P-deta/Ep copolymers inherits the high modulus from P-deta homopolymer. Meanwhile, they also show comparable properties with other $\mathrm{Bz} / \mathrm{Ep}$ binary copolymers [8] and Bz/Ep/amine tertiary copolymers $[14,15]$ in $T_{\mathrm{g}}$, crosslink density and char yield. The thermal stability is worse when P-deta fraction in the copolymers is high.

\section{Conclusions}

This study focuses on an amine-containing Bz prepared from phenol, diethylenetriamine and formaldehyde, its homopolymerization and copolymerization with Ep. The viscosity of P-deta is lower than $1 \mathrm{~Pa} \cdot \mathrm{s}$, which is much lower than BA-a and suitable for low temperature RTM. Polymerization of P-deta undergoes amine-promoted ring-opening, Bz ring-opening and intermediate conversion. However, the conversion is confined to lower than $40 \%$, based on enthalpy calculation. Mixing Ep-with P-deta changes reaction pathways. The copolymerization can be sufficiently proceeded with a faster conversion than P-deta and BA-a. The limit of Bz fraction $(>50 \%)$ and the dilution effect of EP in general Bz/Ep binary system are eliminated in this study. The viscoelastic properties of the homo- and copolymers are also studied. At a 50/50 (wt/wt) ratio, the copolymer exhibits higher $T_{\mathrm{g}}$ than both P-deta homopolymer and Ep cured by DETA. This is quite different from those ternary systems, in which amines were introduced as additives. Accompanied with increasing Ep fraction, the crosslink density is enhanced, while the moduli at room temperature is decreased. Concerning the thermal properties, incorporating more Ep improves the thermal stability of the copolymer, although the char yield becomes less. In conclusion, P-deta homopolymer and P-deta/Ep copolymers show comparable properties with many other reported Bz polymer and copolymer systems, while the liquid phase and low viscosity make it more attractive than those solid $\mathrm{Bz}$ in application.

\section{Acknowledgements}

This research has been supported by the Nansha Innovation Project (2013Z01 3) and Nansha International Cooperation Project (2014GJ01) of Nansha District, Guangzhou, China.

\section{References}

[1] Ning X., Ishida H.: Phenolic materials via ring-opening polymerization of benzoxazines: Effect of molecular structure on mechanical and dynamic mechanical properties. Journal of Polymer Science Part B: Polymer Physics, 32, 1121-1129 (1994).

DOI: $10.1002 /$ polb.1994.090320515

[2] Ishida H., Agag T.: Handbook of benzoxazine resins. Elsevier, Amsterdam (2011).

[3] Zhang L., Zhu Y., Li D., Wang M., Chen H., Wu J.: Preparation and characterization of fully renewable polybenzoxazines from monomers containing multi-oxazine rings. RSC Advances, 5, 96879-96887 (2015). DOI: $10.1039 / \mathrm{c} 5 \mathrm{ra} 17164 \mathrm{~d}$

[4] Xu G-M., Shi T., Liu J., Wang Q.: Preparation of a liquid benzoxazine based on cardanol and the thermal stability of its graphene oxide composites. Journal of Applied Polymers Science, 131, 40353/1-40353/8 (2014). DOI: $10.1002 /$ app.40353

[5] Jubslip C., Takeichi T., Rimdusit S.: Effect of novel benzoxazine reactive diluent on processability and thermomechanical characteristics of bi-functional polybenzoxazine. Journal of Applied Polymer Science, 104, 2928 2938 (2007).

DOI: 10.1002/app.25929

[6] Shi J., Zheng X., Xie L., Cao F., Wu Y., Liu W.: Filmforming characteristics and thermal stability of low viscosity benzoxazines derived from melamine. European Polymer Journal, 49, 4054-4061 (2013).

DOI: $10.1016 /$ j.eurpolymj.2013.09.011

[7] Ishida H., Allen D. J.: Physical and mechanical characterization of near-zero shrinkage polybenzoxazines. Journal of Polymer Science Part B: Polymer Physics, 34, 1019-1030 (1996).

DOI: $10.1002 /($ SICI)1099-0488(19960430)34:6<1019:: AID-POLB1>3.0.CO;2-T

[8] Ishida H., Allen D. J.: Mechanical characterization of copolymers based on benzoxazine and epoxy. Polymer, 37, 4487-4495 (1996).

DOI: $10.1016 / 0032-3861(96) 00303-5$

[9] Uchida S., Kawauchi T., Furukawa N., Takeichi T.: Polymer alloys of high-molecular-weight benzoxazine and epoxy resin. High Performance Polymers, 26, 846-855 (2014).

DOI: $10.1177 / 0954008314532480$

[10] Wang Y-X., Ishida H.: Cationic ring-opening polymerization of benzoxazines. Polymer, 40, 4563-4570 (1999). DOI: 10.1016/S0032-3861(99)00074-9 
[11] Rimdusit S., Ishida H.: Synergism and multiple mechanical relaxations observed in ternary systems based on benzoxazine, epoxy, and phenolic resins. Journal of Polymer Science Part B: Polymer Physics, 38, $1687-$ 1698 (2000).

DOI: $10.1002 / 1099-0488(20000701) 38: 13<1687::$ AIDPOLB20>3.0.CO;2-T

[12] Rimdusit S., Ishida H.: Development of new class of electronic packaging materials based on ternary systems of benzoxazine, epoxy, and phenolic resins. Polymer, 41, 7941-7949 (2000). DOI: $10.1016 / \mathrm{S} 0032-3861(00) 00164-6$

[13] Chow W. S., Grishchuk S., Burkhart T., Karger-Kocsis J.: Gelling and curing behaviors of benzoxazine/epoxy formulations containing 4,4'-thiodiphenol accelerator. Thermochimica Acta, 543, 172-177 (2012). DOI: $10.1016 /$ j.tca.2012.05.015

[14] Rao B. S., Pathak S. K.: Thermal and viscoelastic properties of sequentially polymerized networks composed of benzoxazine, epoxy, and phenalkamine curing agents. Journal of Applied Polymer Science, 100, 3956-3965 (2006).

DOI: $10.1002 /$ app. 23008

[15] Grishchuk S., Mbhele Z., Schmitt S., Karger-Kocsis J.: Structure, thermal and fracture mechanical properties of benzoxazine-modified amine-cured DGEBA epoxy resins. Express Polymer Letters, 5, 273-282 (2011). DOI: 10.3144/expresspolymlett.2011.27

[16] Sun J., Wei W., Xu Y., Qu J., Liu X., Endo T.: A curing system of benzoxazine with amine: Reactivity, reaction mechanism and material properties. RSC Advances, 5, 19048-19057 (2015). DOI: $10.1039 / \mathrm{c} 4 \mathrm{ra} 16582 \mathrm{a}$

[17] Ambrožič R., Šebenik U., Krajnc M.: Novel epoxybenzoxazine water-based emulsions with reactive benzoxazine surfactants for coatings. Express Polymer Letters, 8, 574-587 (2014).

DOI: $10.3144 /$ expresspolymlett.2014.61

[18] Zhao P., Zhou Q., Deng Y. Y., Zhu R. Q., Gu Y.: Reaction induced phase separation in thermosetting/thermosetting blends: Effects of imidazole content on the phase separation of benzoxazine/epoxy blends. RSC Advances, 4, 61634-61642 (2014).

DOI: $10.1039 / \mathrm{c} 4 \mathrm{ra} 10484 \mathrm{f}$

[19] Wang H., Zhao P., Ling H., Ran Q., Gu Y.: The effect of curing cycles on curing reactions and properties of a ternary system based on benzoxazine, epoxy resin, and imidazole. Journal of Applied Polymer Science, 127, 2169-2175 (2013). DOI: 10.1002/APP.37778

[20] Ručigaj A., Alič B., Krajnc M., Šebenik U.: Curing of bisphenol A-aniline based benzoxazine using phenolic, amino and mercapto accelerators. Express Polymer Letters, 9, 647-657 (2015).

DOI: $10.3144 /$ expresspolymlett.2015.60
[21] Agag T., Arza C. R., Maurer F. H. J., Ishida H.: Primary amine-functional benzoxazine monomers and their use for amide-containing monomeric benzoxazines. Macromolecules, 43, 2748-2758 (2010).

DOI: $10.1021 / \mathrm{ma} 902556 \mathrm{k}$

[22] Kawaguchi A. W., Sudo A., Endo T.: Thiol-functionalized 1,3-benzoxazine: Preparation and its use as a precursor for highly polymerizable benzoxazine monomers bearing sulfide moiety. Journal of Polymer Science Part A: Polymer Chemistry, 52, 1448-1457 (2014). DOI: $10.1002 /$ pola.27131

[23] Chaisuwan T., Komalwanich T., Luangsukrerk S., Wongkasemjit S.: Removal of heavy metals from model wastewater by using polybenzoxazine aerogel. Desalination, 256, 108-114 (2010). DOI: $10.1016 /$ j.desal.2010.02.005

[24] Alhwaige A. A., Agag T., Ishida H., Qutubuddin S.: Biobased chitosan/polybenzoxazine cross-linked films: Preparation in aqueous media and synergistic improvements in thermal and mechanical properties. Biomacromolecules, 14, 1806-1815 (2013). DOI: $10.1021 / \mathrm{bm} 4002014$

[25] Ishida H.: Process for preparation of benzoxazine compounds in solventless systems. U.S. Patent 5543516, USA (1996).

[26] Baqar M., Agag T., Huang R., Maia J., Qutubuddin S., Ishida H.: Mechanistic pathways for the polymerization of methylol-functional benzoxazine monomers. Macromolecules, 45, 8119-8125 (2012).

DOI: $10.1021 / \mathrm{ma3} 01963 \mathrm{~d}$

[27] Grishchuk S., Schmitt S., Vorster O. C., Karger-Kocsis J.: Structure and properties of amine-hardened epoxy/ benzoxazine hybrids: Effect of epoxy resin functionality. Journal of Applied Polymer Science, 124, 2824-2837 (2012).

DOI: 10.1002/app.35302

[28] Tobolsky A. V.: Properties and structure of polymers. Wiley, New York, (1962).

[29] Katz D., Tobolsky A. V.: Rubber elasticity in highly crosslinked polyesters. Journal of Polymer Science Part A: Polymer Chemistry, 2, 1587-1594 (1964).

DOI: $10.1002 /$ pol.1964.100020405

[30] Katz D., Tobolsky A. V.: Rubber elasticity in highly crosslinked polyethyl acrylate. Journal of Polymer Science Part A: Polymer Chemistry, 2, 1595-1605 (1964). DOI: $10.1002 /$ pol.1964.100020406

[31] Nielsen L. E.: Mechanical properties of polymers and composites, Vol. I. Marcel Dekker, New York (1983).

[32] Allen D. J., Ishida H.: Effect of phenol substitution on the network structure and properties of linear aliphatic diamine-based benzoxazines. Polymer, 50, 613-626 (2009).

DOI: $10.1016 /$ j.polymer.2008.11.007 